\title{
Breast pathology in Asia
}

Takuya Moriya

Published online: 6 November 2009

(C) The Japanese Breast Cancer Society 2009

The number of breast carcinoma patients has been increasing in Japan. We usually consider the possibility of international comparison studies with western countries, because the incidence of breast cancers among women in these countries is much more frequent than in Japan. Previously, however, there have been few opportunities to compare our data with those from other Asian countries or to characterize the breast lesions of Asian women other than those in Japan.

The 26th Congress of the International Association of Breast Cancer Research (IABCR) was held from September 22-24, 2008, in Kurashiki City, Japan. At the time we invited three experts form Asia to speak during a session on breast pathology. The discussion, and the latest developments in the field, are summarized in this special issue.

Dr. Puay Hoon Tan from Singapore General Hospital introduced us to the current situation of breast cancer in her country, especially epidemiological aspects. The incidence of breast cancer in Singapore is probably the highest among Asian countries, and their analysis will be a useful control when studying the epidemiology in Japanese patients. In addition, I believe that the screening program in Singapore is excellent and it will be a good model for us.

Dr. Gary Tse and his group from Prince of Wales Hospital, Chinese University of Hong Kong, reviewed the pathology of phyllodes tumors. It is not always easy for us to diagnose and grade these tumors pathologically. However, they showed us not only general histological findings but also an immunohistological approach, molecular aspects, and their results of prognosis. Dr. Tse is the foremost among breast pathologists in this field, so our readers should find this review valuable.

Dr. Benjaporn Chaiwun and her colleagues from Chiang Mai University had analyzed clinicopathological features of high nuclear grade breast cancers in Thailand. As many researchers and clinicians are currently very interested in "triple-negative" and "basal-type" breast carcinomas, their study may give us of new information in this field. Triple-negative breast cancer is known to relate to atypical medullary type morphology, but they indicated that triplenegative breast cancers may be a different type or carcinoma from non-triple-negative tumors even in non-medullary carcinomas.

Finally, I would like to express my sincere thanks to my three good friends from Asia for contributing the results of their excellent studies to Breast Cancer. I hope that this special issue may become a nice opportunity to plan new projects or collaborations among Asian groups in the very near future.

T. Moriya ( $₫)$

Department of Pathology, Kawasaki Medical School,

Kurashiki, Japan

e-mail: tmoriya@med.kawasaki-m.ac.jp 